\title{
Estudio Bibliométrico de la Producción Científica sobre la Inspección Educativa
}

\author{
Bibliometric Study of Scientific Production on Educational \\ Inspection
}

\author{
Antonio José Moreno Guerrero \\ Universidad de Granada, España
}

\begin{abstract}
La inspección educativa, como parte de administrativa de la inspección educativa, preserva y defiende la calidad de la educación, siendo un referente para la mejora de la misma. La metodología de investigación aplicada es un análisis bibliométrico a través de los marcadores "Inspección en Educación”, "Inspección educativa”, "Inspección Escolar", "Inspección de la Educación”, "Supervisión educativa”, con un total de 92 documentos analizados. Se han tenido presente el género autor principal, número de autores, año de publicación, institución autores, primer apellido autores, palabras clave, tipo de documento, nombre de la revista, metodología de investigación, población del estudio, instrumentos de investigación, obras más citadas, número de referencias en el artículo, conclusiones investigación y factor de impacto. Se observa que los textos científicos centrados en la inspección educativa son de una autoría, realizado por hombres, aplicando una metodología cualitativa, mediante el análisis de documentos, con referencias inferiores a 20 documentos, siendo el año 2017 el mayor año de producción, con artículos indexados en Latindex y creaciones de textos científicos por parte de la inspección de Andalucía, cuyas palabras más usadas son "Educación" e "Inspección”
\end{abstract}

Descriptores: Inspección educativa; Inspección escolar; Bibliometría; Educación; Calidad de la educación.

\begin{abstract}
The educational inspection, as part of administrative of the educational inspection, preserves and defends the quality of the education, being a reference for the improvement of the same. The methodology of applied research is a bibliometric analysis through the markers "Inspection in Education", "Educational Inspection", "School Inspection", "Inspection of Education", "Educational Supervision", with a total of 92 documents analyzed. The main author gender, number of authors, year of publication, institution authors, first surname authors, key words, type of document, name of the journal, research methodology, study population, research instruments, other works have been taken into account cited, number of references in the article, research conclusions and impact factor. It is observed that the scientific texts focused on the educational inspection are of an authorship, made by men, applying a qualitative methodology, through the analysis of documents, with references inferior to 20 documents, being the year 2017 the biggest year of production, with articles indexed in Latindex and creations of scientific texts by the inspection of Andalusia, whose most used words are "Education" and "Inspection".
\end{abstract}

Keywords: Educational supervision; School supervision; Bibliometrics; Education; Educational quality. 


\section{Revisión de la literatura}

El artículo que se presenta a continuación se fundamenta en un análisis pormenorizado de la producción documental sobre la inspección educativa en el ámbito científico durante los años 2009 y 2019. Para llevar a cabo este análisis se han puesto en práctica diversas técnicas bibliométricas con la intención de analizar la trascendencia a nivel investigativo de la temática tratada.

La finalidad de la investigación es analizar el perfil de los textos científicos sobre la inspección educativa. Los objetivos que se buscan en el artículo son: identificar el número de autores que investigan sobre la temática, determinar el género del autor de referencia, comprobar la metodología de investigación desarrollada, probar las técnicas de recogida de datos que se aplican, esclarecer la evolución en los años de análisis (2009-2019), identificar los autores con mayor producción científica, estipular la frecuencia de palabras usadas tanto los textos como en las palabras clave, comprobar las obras más citadas, así como manifestar el crecimiento exponencial de la producción científica. La inspección educativa es un servicio, aunque necesario e indispensable para asegurar la calidad y equidad educativa, tal y como se establece en la Ley Orgánica 2/2006, de 3 de mayo, de Educación, en su Texto Consolidado, tras su modificación parcial por la Ley Orgánica 8/2013, de 9 de diciembre, para la mejora de la calidad educativa (LOE-TC), es un estamento poco conocido entre la comunidad educativa, al cual recurre cuando presentan problemas concretos (Bolívar, 2018).

La inspección educativa tiene sus inicios con el Estado liberal, entre finales del siglo XVIII y comienzos del siglo XIX (Ramírez, 2017), a posteriori de la Revolución francesa en 1789 (Martínez y Hernández, 2015). Desde entonces hasta la actualidad, esta institución ha sufrido diversos cambios (González, Salmerón y Beas, 2017) que les ha permitido regular su situación hasta la actualidad, de manera más concreta y precisa (Esteban, 2010), aunque ello no ha evitado que tenga tres aspectos divergentes que se consideran como conflictivos en su proceso de actuación, y que en muchos casos los limita en sus acciones, como son (Viñao, 1999): politización-profesionalización; fiscalización-control; y administrativopedagógico. Esto ha generado una diversificación en los distintos modelos de inspección educativa (Choi, 2019).

Tradicionalmente, la función de la inspección de educación ha sido, y lo sigue siendo, la de velar por el cumplimiento de las normas y leyes (Espejo y Calvo, 2015), mediante el control y fiscalización administrativa (Aguerrondo, 2013), tratando de apoyar la función directiva y asesorar a la comunidad educativa (Bolívar, 2018). Dependiendo de su zona de actuación, las funciones varían (Carrasco, 2014), pero de base, todas parten de estas premisas (Álvarez y Pérez, 2010), incluso en Iberoamérica (Cortes y Lorente, 2013), aunque ello no quita que actualmente esté sufriendo una crisis de identidad en las actuaciones que debe desarrollar la propia inspección educativa en su qué hacer diario (Camacho, 2015; Esteban et al., 2016).

La inspección educativa es valorada por los agentes que conforman la comunidad educativa de forma dispar. Según Álvarez y Pérez (2010), la influencia de la inspección de educación en la construcción de la cultura organizativa del propio centro, tanto en la estructura de coordinación como en la documentación oficial, es considerada como un factor relevante, que los propios docentes valoran como muy interesantes. En cambio, ha perdido credibilidad con respecto a la evaluación de centros y como agente de formación, 
debido las tareas o actuaciones burocráticas que desarrollan (Bar y Martínez, 2018), o por las tareas control al entorno docente (Bolívar, 2018). Además, hay quienes consideran que no aporta ningún valor añadido al proceso de enseñanza y aprendizaje (Pérez Aguilar, 2015), y los que valoran que puede contribuir de manera significativa a la innovación educativa en los procesos formativos (Vázquez, 2018). En este aspecto concreto, no hay consenso.

Ello hace que la inspección del sistema educativo requiera de más formación y especialización (Reyzábal, 2015), siendo necesario incluir aspectos innovadores a nivel organizativo y metodológico (Fernández, 2013), para realizar de manera adecuada las funciones que se les atribuyen, y con ello, conseguir su fin, que es mejorar el sistema educativo (Álvarez, Rodríguez y Camacho, 2018). La finalidad es que puedan desarrollar con adecuación la función asesora (Antúnez, 2009), y hacer frente a los nuevos modelos de regulación administrativa (Cuadrado, 2018), y a los nuevos tiempos que se presentan (García y Pérez, 2017). Esa formación debe llevar una mejora en las distintas herramientas que usa la inspección educativa para recopilar y transmitir la información (Pérez Aguilar, 2015). Por ello, se puede considerar la inspección educativa como un servicio público esencial y de calidad que vela por el sistema educativo (Esteban, 2011), siendo un actor fundamental en la innovación de los procesos de enseñanza y aprendizaje (Estefanía, 2017), promoviendo cambios educativos (Silva, 2013), garantizando con ello el derecho de las personas que conforman la comunidad educativa (Luzarraga, Núñez y Etxeberría, 2018).

Dada la importancia dentro del ámbito educativo, y con la finalidad de ofrecer una base a la que acudir para la realización de un estudio en el ámbito de la inspección educativa, se plantean las siguientes cuestiones: ¿cuántos autores suelen desarrollar investigaciones sobre la inspección educativa?, ¿cuál es el género de los investigadores?, ¿qué metodología de investigación se sigue?, ¿cuáles son las principales técnicas de recogidas de datos?, ¿Cómo ha evolucionado la investigación en inspección educativa?, ¿cuáles son los autores con más producción científica?, ¿cuál es la frecuencia de palabra que se da en estos textos científicos?, etc. Estas y otras cuestiones serán respondidas a lo largo de este documento, tratando de dar una visión general a aquellos investigadores que deseen orientar sus estudios a la inspección educativa.

\section{Método}

La metodología de estudio aplicada es de tipo bibliométrico (Rodríguez, Trujillo y Sánchez, 2019), mediante una evaluación de rendimiento de la producción científica (Cobo et al., 2011). El artículo parte de las premisas marcadas por Anta (2008) y Álvarez (2015) sobre las técnicas de rastreo, analítica y de cuantificación documental a desarrollar en el análisis bibliométrico, además de incluir otros indicadores necesarios para el adecuado desarrollo de la investigación (Gómez et al., 2012).

El análisis bibliométrico se fundamenta en un método cuantitativo, de tipo descriptivo de toda la producción científica (Davis y González, 2003), en consonancia con la inspección educativa, analizando la producción científica producida entre los años 2009-2019, ambos inclusive. El proceso de localización documental se desarrolló en el buscador Google Scholar, el cual está destinado y especializado en la búsqueda de textos científicos (Torres, Ruiz y Delgado, 2009), en el que aparecen otras bases de datos que contienen revistas 
indizadas en Scopus, Web of Science, Scielo, Redalyc, Latindex y Dialnet, entre otras. El registro documental se ha desarrollado mediante el formulario de Google, siguiendo de modelo una matriz de protocolo PRISMA-P (Moher et al., 2015), con las siguientes variables de búsqueda: Género autor principal; Número de autores; Año de publicación; Institución autores; Primer apellido autores; Palabras clave; Tipo de documento; Nombre de la revista; Metodología de investigación; Población del estudio; Instrumentos de investigación; Número de referencias en el artículo; Conclusiones investigación, y Factor de impacto. En todas estas variables, se tuvo presente la documentación que hacía referencia directa a la Inspección Educativa desde la perspectiva de la supervisión educativa.

La unidad de análisis de todos los documentos encontrados, un total de 8104 publicaciones de diversa índole, tras la aplicación de diversas ecuaciones de búsqueda concreta sobre los términos en los que se centró la investigación, en enero de 2019, teniendo de referencia los tesauros: UNESCO, Europeo de la Educación y ERIC. Las palabras claves obtenidas en la búsqueda de los tesauros permitió establece los siguientes marcadores y operadores booleanos (Aleixandre et al., 2011) de búsqueda tanto en el cuerpo del documento como en el título: "Inspección en Educación" ( $\mathrm{N}=12)$ [Title/Abstract/Text] OR "Inspección educativa" $(\mathrm{N}=2460)$ [Title/Abstract/Text $]$ OR "Inspección Escolar" $(\mathrm{N}=752)$ [Title/Abstract/Text $]$ OR "Inspección de la Educación" (N = 120) [Title/Abstract/Text] OR "Supervisión educativa" $(\mathrm{N}=4760)$ [Title/Abstract/Text] OR "Supervisión centros educativos" $(\mathrm{N}=0)[$ Title/Abstract/Text $]$.

El total de documentos encontrados $(\mathrm{N}=8140)$ fue sometido a una evaluación previa para descartar aquellos escritos científicos que no tenían relación con la investigación centrada en la inspección educativa, ya que se ajustaban a un procedimiento pedagógico en el que conllevaba la aprobación de la inspección educativa, en legislación citada en el marco teórico, o en textos científicos donde los propios inspectores de educación han elaborado artículos científicos sobre otras temáticas. Para dicha tarea, hicimos uso del software de tratamiento de textos NVivo 11, aplicando la frecuencia de palabras que ofrece el programa en los documentos descargados, y mostrando los resultados en texto amplio, permitiendo ver y analizar cada uno de los párrafos donde se establecía las palabras claves indicadas con anterioridad. De esta manera la muestra quedó en un total de 92 publicaciones de diversa índole.

Además, se han utilizado indicadores bibliométricos de tipo personal, de producción de forma y contenidos para facilitar el análisis de los diversos resultados (Velasco, Eiros y San Román, 2012), y poder contabilizar eficazmente los documentos encontrados en la literatura (González et al., 2012; Maltrás, 2003).La recogida de datos se desarrolló mediante el formulario de Google para facilitar la recopilación de los datos, para su posterior análisis mediante los programas de análisis de datos IBM SPSS 20, para los parámetros cuantitativos, y NVivo 11, para los parámetros más cualitativos. En el proceso de análisis de los resultados, se tuvieron en cuenta la ley de Lotka o productividad de los autores (Urbizagástegui, 2005) y la ley de Price o del crecimiento exponencial de las obras producidas (Price, 1986). 


\section{Resultados}

Los resultados que aquí se presentan hacen referencia a los 92 documentos analizados después la búsqueda bibliográfica. Dada la gran cantidad de variables analizadas, se presentan en pequeños apartados: opciones de búsqueda, género primer autor, número de autores, metodología de investigación, técnicas de recogidas de datos, referencias bibliográficas, año de publicación, indexaciones, procedencias de los archivos, autores con más producción científica, instituciones, tipo de documento, frecuencia de palabras claves, frecuencia de palabras de la producción científica, relación entre año de publicación y género del primer autor, documentos más citados, relación entre año de publicación y número de autores, ley de Lotka y crecimiento exponencial de la producción científica.

Resultados obtenidos mediante las opciones de búsqueda

De las opciones de búsqueda establecida para el análisis de la inspección educativa en los textos científicos, podemos observar que IE presenta un mayor peso que el resto, seguido de SE, INES e INED. Ello muestra que las investigaciones sobre esta temática se fundamentan en inspección educativa (cuadro 1).

Cuadro 1. Resultados obtenidos de las opciones de búsqueda

\begin{tabular}{lcc}
\hline \multicolumn{1}{c}{ PALABRA BÚSQUEDA } & REFERENCIAS & \% DE 92 \\
\hline Inspección de la educación (INED) & 4 & 4,3 \\
Inspección escolar (INES) & 11 & 12,0 \\
Inspección educativa (IE) & 56 & 60,9 \\
Supervisión educativa (SE) & 21 & 22,8 \\
\hline
\end{tabular}

Fuente: Elaboración propia.

\section{Análisis de la producción científica en función al género del primer autor}

Comenzando con el análisis de las diversas variables planteadas durante el desarrollo, los datos, marcados en el cuadro 2 , muestran una mayor incidencia de los hombres que las mujeres, de forma considerable, en la temática de investigación centrada en la inspección educativa. Se establece una pequeña diferencia cuando resultado de búsqueda es INES, en la que se da el caso contrario, siendo las mujeres las que publican más que los hombres.

Cuadro 2. Resultados obtenidos de la producción científica en función al género del primero autor

\begin{tabular}{lcccccc}
\hline & INED & INES & IE & SE & TOTAL/AÑO & \% DE 92 \\
\hline Hombre & 4 & 5 & 45 & 12 & 66 & 71,7 \\
Mujer & 0 & 6 & 11 & 9 & 26 & 28,3 \\
\hline Total & 4 & 11 & 56 & 21 & 92 & 100 \\
\hline
\end{tabular}

Fuente: Elaboración propia.

\section{Análisis de la producción científica en función al número de autores por documento}

El número de textos científicos sobre esta temática es elaborado principalmente por un solo autor, con gran diferencia sobre los textos en los que tienen dos autores o más. En las búsquedas de INEDe INES, no hay textos científicos de 4 autores o más autores (cuadro $3)$. 
Análisis de la producción científica en función a la metodología de investigación desarrollada

La metodología aplicada principalmente para el desarrollo de las investigaciones centradas en esta temática es cualitativa, de manera muy destacada sobre el resto de metodologías de investigación. Las pocas investigaciones cuantitativas o mixtas se encuentran en las búsquedas realizadas en IE y SE, según se observa en el cuadro 4.

Cuadro 3. Resultados obtenidos de la producción científica en función al número de autores

\begin{tabular}{lcccccc}
\hline & INED & INES & IE & SE & TOTAL/AÑO & \% DE 92 \\
\hline 1 autor & 2 & 10 & 37 & 10 & 59 & 64,1 \\
2 autores & 2 & 0 & 10 & 7 & 19 & 20,7 \\
3 autores & 0 & 1 & 7 & 2 & 9 & 9,8 \\
4 autores & 0 & 0 & 1 & 1 & 3 & 3,3 \\
Más de 4 autores & 0 & 0 & 1 & 2 & 2 & 2,2 \\
\hline Total & 4 & 11 & 56 & 21 & 92 & 100 \\
\hline
\end{tabular}

Fuente: Elaboración propia.

Cuadro 4. Resultados obtenidos de la producción científica en función a la metodología de investigación

\begin{tabular}{lcccccc}
\hline & INED & INES & IE & SE & TOTAL/AÑO & \% DE 92 \\
\hline Cuantitativa & 0 & 0 & 4 & 3 & 7 & 7,6 \\
Cualitativa & 4 & 11 & 51 & 18 & 84 & 91,3 \\
Mixta & 0 & 0 & 1 & 0 & 1 & 1,1 \\
\hline Total & 4 & 11 & 56 & 21 & 92 & 100 \\
\hline
\end{tabular}

Fuente: Elaboración propia.

Análisis de la producción científica en función a los instrumentos o técnicas de recogida de datos

El instrumento utilizado para las investigaciones centradas en la temática de Inspección Educativa es el documento y la propia legislación educativa, dado que los estudios se centran en la revisión bibliográfica. Se utilizan otros instrumentos (cuestionario, entrevista o historia de vida) en las búsquedas de IE y SE, siendo inexistente en INED e INES, tal y como se puede observar en el cuadro 5 .

Cuadro 5. Resultados obtenidos de la producción científica en función al instrumento de investigación

\begin{tabular}{lcccccc}
\hline & INED & INES & IE & SE & TOTAL/AÑo & \% DE 92 \\
\hline Cuestionario & 0 & 0 & 5 & 2 & 7 & 7,6 \\
Documento & 4 & 11 & 44 & 18 & 77 & 83,7 \\
Entrevista & 0 & 0 & 5 & 1 & 6 & 6,5 \\
Historia de vida & 0 & 0 & 2 & 0 & 2 & 2,2 \\
\hline Total & 4 & 11 & 56 & 21 & 92 & 100 \\
\hline
\end{tabular}

Fuente: Elaboración propia.

Análisis de la producción científica en función al número de referencias bibliográficas de los documentos

Los documentos científicos analizados tienen pocas referencias, situándose la mayoría de ellos por debajo de 20. Son pocos los documentos que recogen más de 40 referencias en sus estudios, localizándose en los textos científicos en las búsquedas de IE y SE (cuadro 6). 
Análisis de la producción científica en función al año de publicación

Según se establece en el cuadro 7, la producción científica tiene su mayor incidencia en los años 2014, 2017 y 2018, y una menor en los años 2010, 2012 y, actualmente, en 2019, aunque esto último puede deberse a que el año acaba de empezar.

Cuadro 6. Resultados obtenidos de la producción científica en función al número de referencias

\begin{tabular}{lcccccc}
\hline & INED & INES & IE & SE & TOTAL/AÑO & \% DE 92 \\
\hline Hasta 20 & 1 & 7 & 32 & 13 & 53 & 57,6 \\
Entre 21 y 40 & 3 & 4 & 18 & 5 & 30 & 32,6 \\
Más de 40 & 0 & 0 & 6 & 3 & 9 & 9,8 \\
\hline Total & 4 & 11 & 56 & 21 & 92 & 100 \\
\hline
\end{tabular}

Fuente: Elaboración propia.

Cuadro 7. Resultados obtenidos de la producción científica en función al año de publicación

\begin{tabular}{lcccccc}
\hline & INED & INES & IE & SE & TOTAL/AÑO & \% DE 92 \\
\hline 2009 & 0 & 2 & 3 & 0 & 5 & 5,4 \\
2010 & 0 & 0 & 2 & 0 & 2 & 2,2 \\
2011 & 0 & 0 & 4 & 3 & 7 & 7,6 \\
2012 & 1 & 0 & 1 & 1 & 3 & 3,3 \\
2013 & 0 & 1 & 4 & 2 & 7 & 7,6 \\
2014 & 1 & 2 & 12 & 0 & 15 & 16,3 \\
2015 & 0 & 2 & 5 & 4 & 11 & 12,0 \\
2016 & 1 & 2 & 4 & 3 & 10 & 10,9 \\
2017 & 0 & 2 & 8 & 6 & 16 & 17,4 \\
2018 & 0 & 0 & 13 & 2 & 15 & 16,3 \\
2019 & 1 & 0 & 0 & 0 & 1 & 1,1 \\
\hline Total & 4 & 11 & 56 & 21 & 92 & 100 \\
\hline
\end{tabular}

Fuente: Elaboración propia.

En la figura 1 se observa de forma visual la evolución de la producción científica en la inspección educativa.

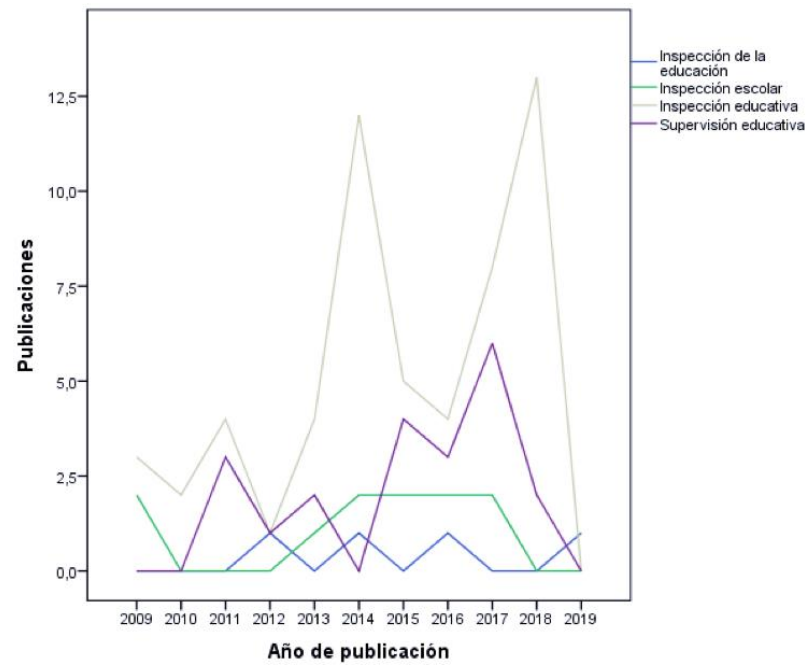

Figura 1. Relación entre número de producciones científicas y año de publicación Fuente: Elaboración propia. 


\section{Base de datos publicaciones}

Tal y como se observa en el cuadro 8 , los documentos analizados, además de la base de datos de Google Scholar, se encuentran albergados principalmente en Latindex, habiendo poca producción en el resto de base de datos. Destaca la alta presencia de textos científicos que no está recogidos en ninguna de las bases de datos analizadas.

Cuadro 8. Resultados obtenidos de la producción científica en función a la indexación

\begin{tabular}{lcccccc}
\hline & INED & INES & IE & SE & TOTAL/AÑ & \% DE 92 \\
\hline Latindex & 2 & 7 & 44 & 12 & 65 & 70,7 \\
Scopus & 0 & 0 & 2 & 3 & 5 & 5,4 \\
JCR & 0 & 0 & 1 & 0 & 1 & 1,1 \\
WOS & 0 & 1 & 1 & 0 & 2 & 2,2 \\
No indexada & 2 & 3 & 8 & 6 & 19 & 20,7 \\
\hline Total & 4 & 11 & 56 & 21 & 92 & 100 \\
\hline
\end{tabular}

Fuente: Elaboración propia.

\section{Procedencia de los archivos}

La producción periódica producida sobre la temática relacionada con la inspección educativa se recoge principalmente en la revista de la Asociación de Inspectores de Educación de España, seguida a una distancia considerable, la revista del Fórum de Aragón. El resto de producciones se encuentran en una horquilla de entre 3 y 1. Además, se centra en las búsquedas IE y SE, tal y como podemos observar en el cuadro 9.

Cuadro 9. Publicaciones periódicas que más han producido sobre inspección educativa

\begin{tabular}{|c|c|c|c|c|c|c|}
\hline & INED & INES & IE & $\mathrm{SE}$ & TOTAL/AÑo & \% DE 92 \\
\hline $\begin{array}{l}\text { Revista de la Asociación de } \\
\text { Inspectores de Educación de } \\
\text { España }\end{array}$ & 2 & 3 & 35 & 1 & 41 & 44,6 \\
\hline Fórum Aragón & $\mathrm{O}$ & $\mathrm{O}$ & 10 & 1 & 11 & 11,9 \\
\hline Educar & $\mathrm{O}$ & $\mathrm{O}$ & 6 & 2 & 8 & 8,6 \\
\hline $\begin{array}{l}\text { REICE. Revista Iberoamericana } \\
\text { sobre Calidad, Eficacia y } \\
\text { Cambio en Educación }\end{array}$ & $\mathrm{O}$ & $\mathrm{O}$ & $\mathrm{O}$ & 3 & 3 & 3,3 \\
\hline Supervisión 21 & $\mathrm{O}$ & $\mathrm{O}$ & 2 & 1 & 3 & 3,3 \\
\hline Total & 2 & 3 & 40 & 8 & 66 & 71,7 \\
\hline
\end{tabular}

Fuente: Elaboración propia.

\section{Autores con más producción científica sobre la temática}

Los autores que más investigan sobre la inspección educativa son Pérez Aguilar, el cual tiene una alta producción en el campo, seguido de Vázquez Cano, Silva García, Esteban Frades y Camacho Prats. Tal y como se puede comprobar en el cuadro 10, los autores centran su búsqueda en IE y SE.

\section{Instituciones}

La institución de los autores que más producción científica desarrollan sobre la temática se centra principalmente en la Inspección de Andalucía, seguida de lejos por la Universidad de Barcelona, Inspección de Madrid, Universidad de Granada y UNED. Es reseñable que la temática de búsqueda de la Inspección de Andalucía se centre en la IE (cuadro 11). 
Cuadro 10. Autores con más producción científica sobre inspección educativa

\begin{tabular}{lcccc}
\hline & IE & SE & TOTAL & \% DE 92 \\
\hline Pérez Aguilar, José Francisco & 8 & 0 & 8 & 8,6 \\
Camacho Prats, Alexander & 6 & 0 & 6 & 6,5 \\
Vázquez Cano, Esteban & 4 & 2 & 6 & 6,5 \\
Esteban Frades, Santiago & 5 & 0 & 5 & 5,4 \\
Silva García, Patricia & 4 & 1 & 5 & 5,4 \\
Antúnez Marcos, Serafín & 2 & 1 & 3 & 32 \\
Aguerrondo, Inés & 0 & 2 & 2 & 2,2 \\
Bolívar Botía, Antonio & 2 & 0 & 2 & 2,2 \\
Romero García, Miguel Ángel & 2 & 0 & 2 & 2,2 \\
Tamayo Pupo, Arismendis & 0 & 2 & 2 & 2,2 \\
Luna Ariza, Pedro Ángel & 2 & 0 & 2 & 2,2 \\
\hline Total & 22 & 5 & 27 & 29,3 \\
\hline
\end{tabular}

Fuente: Elaboración propia.

Cuadro 11. Instituciones con mayor producción científica

\begin{tabular}{lcccccc}
\hline & INED & INES & IE & SE & TOTAL/AÑO & \% DE 92 \\
\hline Inspección Andalucía & 0 & 0 & 17 & 0 & 17 & 18,5 \\
Universidad de Barcelona & 1 & 2 & 2 & 0 & 5 & 5,4 \\
Inspección Madrid & 0 & 0 & 5 & 0 & 5 & 5,4 \\
Universidad de Granada & 0 & 0 & 3 & 0 & 3 & 3,3 \\
UnED & 0 & 1 & 1 & 2 & 4 & 4,3 \\
\hline Total & 1 & 2 & 28 & 2 & 33 & 36,9 \\
\hline
\end{tabular}

Fuente: Elaboración propia.

\section{Tipo de documento}

El tipo de documento que más recoge las investigaciones sobre la inspección educativa son los artículos científicos, seguidos muy de lejos por los capítulos de libros, tal y como se observa en el cuadro 12.

Cuadro 12. Resultados obtenidos de la producción científica en función al tipo de documento

\begin{tabular}{lcccccc}
\hline & INED & INES & IE & SE & TOTAL/AÑO & \% DE 92 \\
\hline Artículo & 4 & 9 & 53 & 18 & 84 & 91,3 \\
Capítulo de libro & 0 & 1 & 2 & 2 & 5 & 5,4 \\
Libro & 0 & 1 & 0 & 1 & 2 & 2,2 \\
Otros & 0 & 0 & 1 & 0 & 1 & 1,1 \\
\hline Total & 4 & 11 & 56 & 21 & 92 & 100 \\
\hline
\end{tabular}

Fuente: Elaboración propia.

\section{Frecuencia de palabras clave en los documentos analizados}

La frecuencia de palabras clave en los 92 documentos analizados sobre la inspección educativa muestra que las palabras más repetidas en ellos son inspección, educación, evaluación, formación y competencias, marcando así el perfil de las investigaciones sobre la temática (cuadro 13). 
Cuadro 13. Palabras clave más frecuentemente usadas en los textos científicos sobre inspección educativa

\begin{tabular}{lcc}
\hline & CONTEO & PORCENTAJE PONDERADO (\%) \\
\hline Inspección & 47 & 4,83 \\
Educación & 40 & 4,11 \\
Evaluación & 27 & 2,77 \\
Formación & 23 & 2,36 \\
Competencias & 20 & 2,05 \\
Supervisión & 18 & 1,85 \\
\hline
\end{tabular}

Fuente: Elaboración propia.

Frecuencias de palabras en los documentos analizados

En relación a las palabras más frecuentes utilizadas en los documentos científicos, se observa que las palabras educación, inspección, educativa, formación y centros son las más repetidas, con una incidencia bastante alta, tal y como se observa en el cuadro 14 .

Cuadro 14. Las 10 palabras más frecuentemente usadas en los textos científicos sobre inspección educativa

\begin{tabular}{lcc}
\hline & CONTEO & PORCENTAJE PONDERADO (\%) \\
\hline Educación & 10383 & 0,51 \\
Inspección & 8480 & 0,42 \\
Educativa & 7402 & 0,37 \\
Formación & 4558 & 0,23 \\
Centros & 4152 & 0,21 \\
Evaluación & 3846 & 0,19 \\
Supervisión & 3693 & 0,18 \\
Revista & 3223 & 0,16 \\
Desarrollo & 3169 & 0,16 \\
Inspectores & 2981 & 0,15 \\
Sistema & 2843 & 0,14 \\
\hline
\end{tabular}

Fuente: Elaboración propia.

Un ejemplo gráfico, de las palabras más repetidas en los textos científicos sobre inspección educativa, se pueden localizar en la siguiente nube de palabras (figura 2).

\section{Relación entre año de publicación género del primer autor}

Tal y como se observa en la figura 3, se establece como aspecto significativo el pico de bajada de producción científica realizado por parte de los hombres en 2015 y la subida de producción de las mujeres ese mismo año. Además, se observa una tendencia un poco ascendente por parte de las mujeres durante los años 2015 y 2017. 


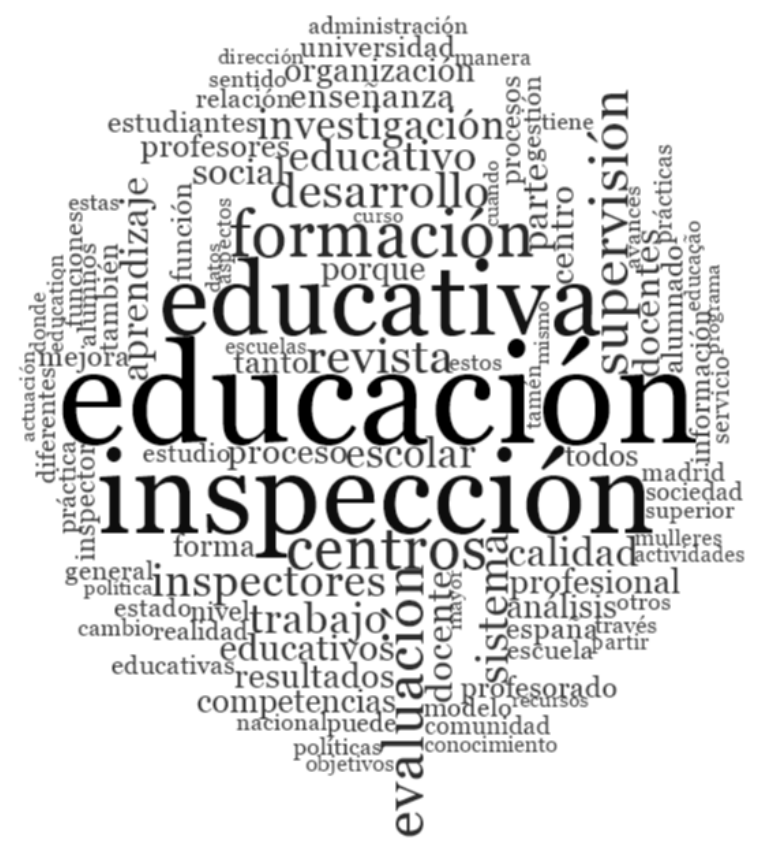

Figura 2. Frecuencias de palabras en los escritos científicos sobre inspección educativa Fuente: Elaboración propia.

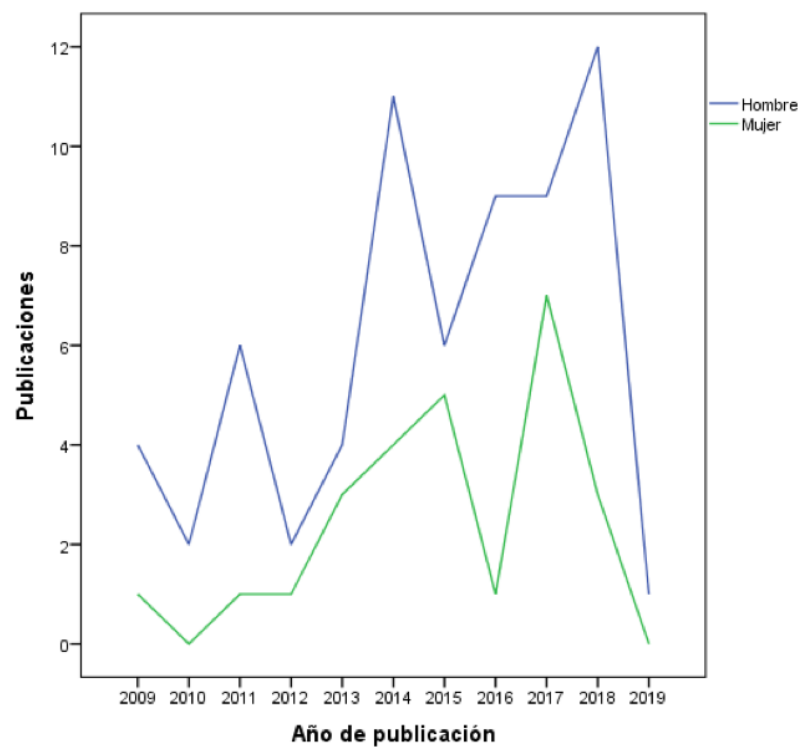

Figura 3. Relación entre año de publicación y género del primer autor Fuente: Elaboración propia.

\section{Referencias más citadas}

El autor más citado en la temática de estudio es Soler (1993), seguido de cerca por Martín (1988), siendo ambas obras libros. El artículo con más citas es el de Antúnez (2009). El resto de documentos presentan menos de 20 citas (cuadro 15). 
Cuadro 15. Referencias más citadas en la producción científica

\begin{tabular}{|c|c|c|c|c|}
\hline Autores & AÑo & TíTUlo & $\begin{array}{c}\text { FUENTE DE } \\
\text { PROCEDENCIA }\end{array}$ & $\begin{array}{l}\text { NÚMERO } \\
\text { DE CITAS }\end{array}$ \\
\hline Soler & 1993 & Fundamentos de supervisión educativa & $\begin{array}{l}\text { Editorial la } \\
\text { Muralla }\end{array}$ & 43 \\
\hline Martín & 1988 & Supervisión educativa & Editorial UNED & 37 \\
\hline Antúnez & 2009 & $\begin{array}{l}\text { La inspección educativa y la evaluación } \\
\text { de la formación permanente de los } \\
\text { profesionales de la educación }\end{array}$ & $\begin{array}{l}\text { Avances en } \\
\text { Supervisión } \\
\text { Educativa }\end{array}$ & 24 \\
\hline Aguerrondo & 2013 & $\begin{array}{l}\text { El rol de la supervisión educativa en la } \\
\text { gestión de las políticas públicas }\end{array}$ & Educar & 18 \\
\hline Muñoz & 1991 & La supervisión educativa & Bordón & 17 \\
\hline Viñao & 1999 & $\begin{array}{l}\text { La inspección educativa. Análisis socio- } \\
\text { histórico de una profesional }\end{array}$ & Bordón & 17 \\
\hline Pavón & 2010 & $\begin{array}{l}\text { La supervisión educativa para la sociedad } \\
\text { del conocimiento: Actualización y } \\
\text { formación }\end{array}$ & $\begin{array}{l}\text { Editorial la } \\
\text { Muralla }\end{array}$ & 15 \\
\hline Silva & 2013 & $\begin{array}{l}\text { El papel de la inspección escolar en la } \\
\text { mejora de los resultados educativos }\end{array}$ & Educar & 14 \\
\hline Esteban & 2007 & $\begin{array}{l}\text { Reflexiones sobre las antonimias de la } \\
\text { inspección educativa en España. Un } \\
\text { problema sin resolver }\end{array}$ & $\begin{array}{l}\text { Avances en } \\
\text { Supervisión } \\
\text { Educativa } \\
\end{array}$ & 12 \\
\hline $\begin{array}{l}\text { Cortés y } \\
\text { Lorente }\end{array}$ & 2011 & $\begin{array}{l}\text { La supervisión educativa en América } \\
\text { Latina antes de las Metas Educativas de } \\
\text { 2021, propuesta por la OEI }\end{array}$ & $\begin{array}{l}\text { Revista } \\
\text { Iberoamericana } \\
\text { de Educación }\end{array}$ & 12 \\
\hline
\end{tabular}

Fuente: Elaboración propia.

\section{Relación entre año de publicación y número de autores}

El número de autores que ha realizado investigaciones sobre la inspección educativa ha sido mayoritariamente de uno, pero se vislumbra un pequeño cambio de tendencia en los últimos años, donde los documentos elaborado por 3 autores están ascendiendo tímidamente, tal y como se establece en la figura 4.

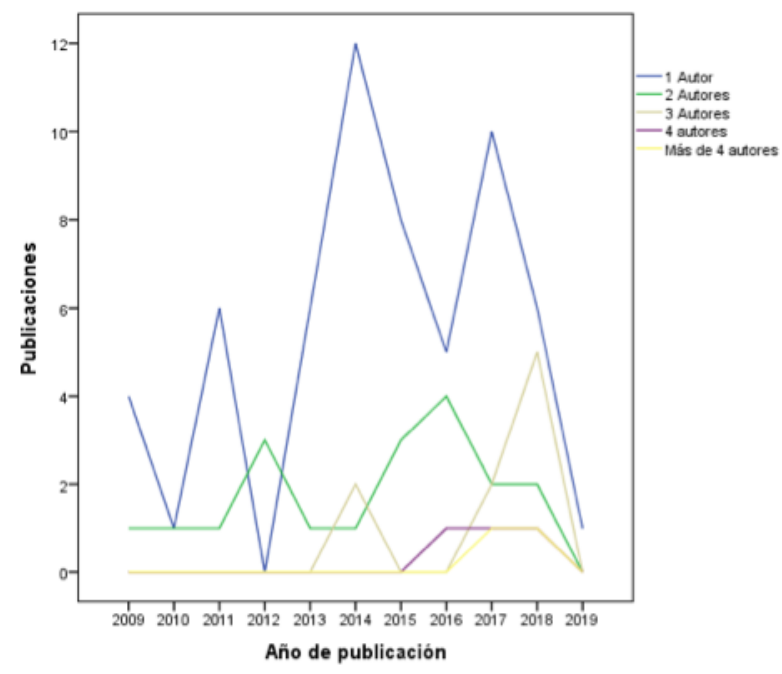

Figura 4. Relación entre año de publicación y número de autores

Fuente: Elaboración propia. 


\section{Ley de Lotka}

En este caso, tal y como se refleja en la figura 5, sobre la productividad personal, la ley de Lotka establece que gran parte de las contribuciones científicas son provenientes de un número reducido de autores, confirmado en la correlación positiva alcanzada entre el menor número de autores y el mayor número de publicaciones efectuadas. Esta premisa, en el artículo presentado, se confirma, ya que la correlación entre el menor número de autores y el mayor número de trabajos es positiva $\left(\mathrm{R}^{2}=0,9812\right)$.

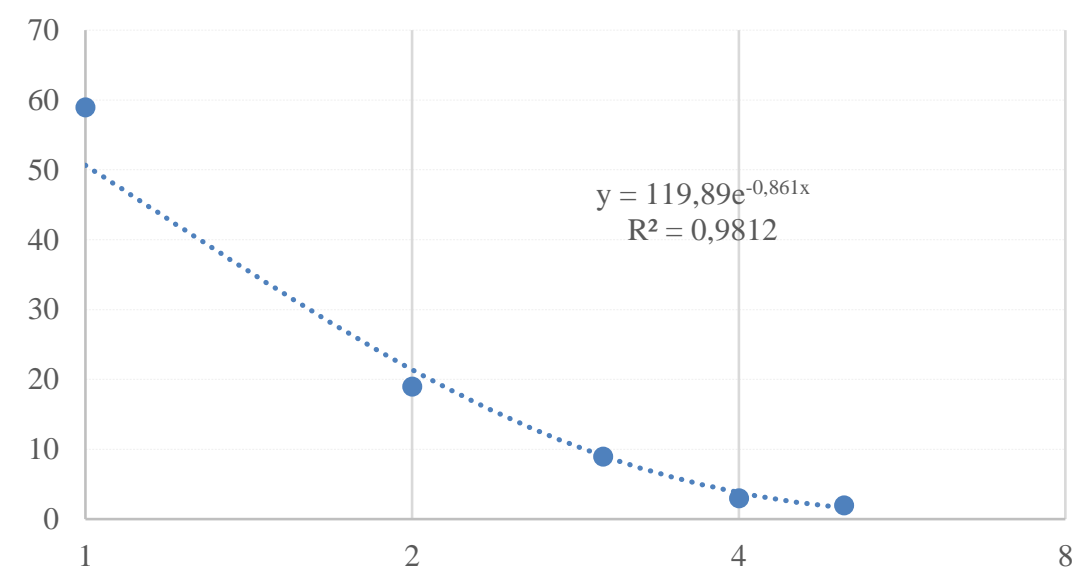

Figura 5. Ley de Lotka en la producción científica sobre la Inspección educativa Fuente: Elaboración propia.

\section{Crecimiento exponencial de la producción científica}

El crecimiento exponencial de la literatura reportada, representada en la figura 6, muestra tres repuntes claramente diferenciados, uno en el año 2011, otro en 2014 y finalmente en 2017. Esto muestra que la tendencia los próximos años puede ser descendente, para volver a retomar su ascenso pasado un par de años. La propensión, a rasgos generales, ha sido ascendente durante el periodo de años analizado.

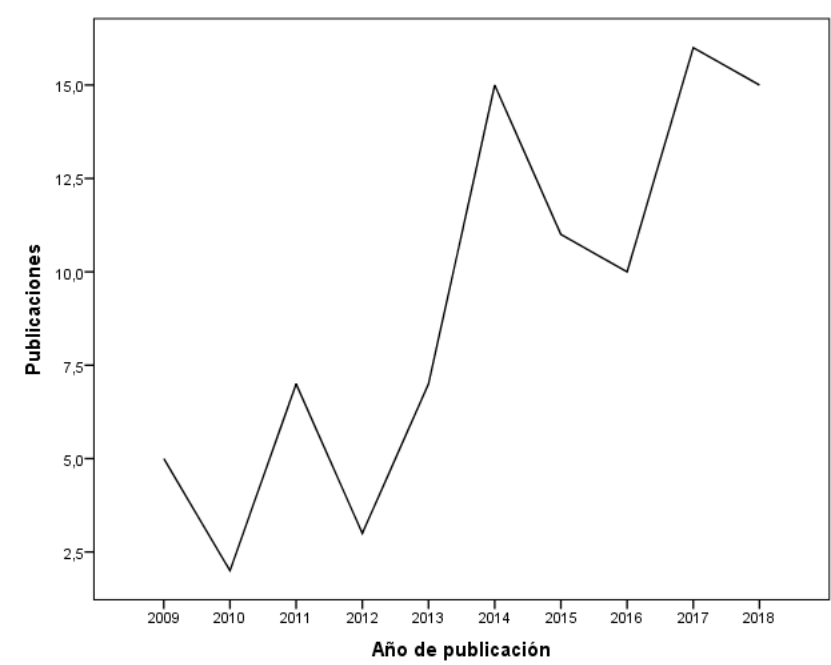

Figura 6. Crecimiento exponencial de la producción científica Fuente: Elaboración propia. 


\section{Discusión y conclusiones}

En la presentación de los resultados se han presentado los datos obtenidos de las preguntas planteadas al inicio sobre la investigación. En principio, la producción científica con las variables "Inspección de la educación (INED)", "Inspección escolar (INES)", "Inspección educativa (IE)" y "Supervisión educativa (SE)" era bastante alta, considerándose con un alto impacto en la producción científica, pero luego, gracias al programa Nvivo 11, se comprobó que la producción que atendía directamente a las investigaciones centradas sobre la inspección educativa bajaba considerablemente, dado que la mayoría de los textos hacia uso de las variables de búsqueda para explicar un procedimiento específico, en el que la inspección educativa era requisito indispensable, no siendo centro de investigación.

Aun así, hay una alta producción de investigaciones sobre la temática, tanto en el ámbito nacional e internacional en relación a la de inspección educativa (Ozkoidi y Albéniz, 2014; Secadura, 2011), llevándose a cabo publicaciones en revistas especializadas y estudios a través de manuales (López, 2014).

La Inspección Educativa es considerada actualmente un elemento de calidad y equidad (Esteban, 2011), que incide de la mejora del sistema educativo (Álvarez, Rodríguez y Camacho, 2018), y siendo un actor fundamental en la innovación de los procesos de enseñanza y aprendizaje (Estefanía, 2017). El hecho de investigar sobre el estamento de la Inspección Educativa se hace necesario para analizar sus actuaciones y proponer actuaciones que sirvan para su verdadero fin, que es la mejora del sistema educativo y los resultados de los procesos de enseñanza y aprendizaje.

En el desarrollo del texto se ha presentado los resultados por apartados, para hacer más visible las distintas variables de investigación que se han planteado. Dicho esto, se mostrarán las deducciones más relevantes encontradas, para luego establece el perfil actual en el que se encuentra la Inspección Educativa.

La producción científica tiene su mayor incidencia en la Inspección Educativa (IE), con un $60.9 \%$, siendo un referente adecuado para la búsqueda de textos científicos sobre la temática. Es cierto, que el término de Supervisión Educativa (SE), aunque es usado en el ámbito europeo, tiene mayor incidencia en la zona de Latinoamérica.

Los textos generados desde el año 2009 suman un total de 92, lo que hace una media aproximada de 10 textos por años, aunque como se puede establecer, la producción se acumula en los años 2014 (16,3\%), 2017 (17,4\%) y 2018 (16,3\%), habiendo una tendencia ascendente en los últimos tiempos, lo que nos sugiere que la temática centrada en la inspección educativa está generando más inquietudes entre los investigadores.

El género del primer autor de las producciones científicas es un aspecto destacable, dado que los hombres tienen una mayor incidencia $(71,7 \%)$ que las mujeres en este campo de investigación. En la relación establecida entre el género del autor de referencia y los años de producción, se muestran picos ascendentes por parte de las mujeres que investigan sobre la temática durante los años 2015 y 2017, aunque no llegan a superar a los hombres en producción científica.

También lo es el número de autores, donde destaca la autoría de solitario (64,1\%) durante el periodo analizado, aunque dicha tendencia está cambiando en los últimos años. Así como 
lo es el número de referencias bibliográficas usadas en las producciones científicas, que está por debajo de $20(57,6 \%)$.

La metodología de investigación es principalmente cualitativa, centrada en el análisis de documentación especializada en el tema y en el análisis de la legislación propiamente educativa. No se observa una producción de relevancia con técnicas cuantitativas o mixtas, lo que abre una puerta de investigación en el futuro para aquellos que quieran iniciar sus estudios en la temática.

Las bases de datos donde se encuentran indexadas las investigaciones es principalmente Latindex (70,7\%), en las que se localizan las dos principales revistas que reciben y publican sobre la temática, como son Revista de la Asociación de Inspectores de Educación de España y Fórum de Aragón. También se encuentran las revistas Supervisión 21 y REICE. Revista Iberoamericana sobre Calidad, Eficacia y Cambio en Educación, aunque esta última está indexada en Scopus. Este hecho se produce debido a que la principal tipología del documento son los artículos de investigación (91,3\%), situados muy por encima del resto de escritos.

El autor que más escribe sobre la temática es Pérez Aguilar, que es Inspector de Educación, destacado de forma considerable con respecto al resto de autores, entre los que destacan Vázquez Cano, Esteban Frades, Silva García y Camacho Prats. Las instituciones de los autores se reparten entre Inspecciones educativas de diversas comunidades autónomas, entre la que lidera Andalucía $(18,5)$, y Universidades, entre las que se encuentran la Universidad de Barcelona y la UNED. Las obras más citadas son de Soler (1993), Martín (1988) y Antúnez (2009).

Analizados los propios textos, se observa que las palabras clave que se usan con más frecuencia son "Inspección", "Educación", "Evaluación", "Formación”, "Competencias" y "Supervisión”, mientras que las palabras más utilizadas en el desarrollo de los textos son "Educación”, "Inspección”, "Educativa”, "Formación”, "Centros", "Evaluación”, "Supervisión”, "Revista”, "Desarrollo", "Inspectores" y "Sistema”. Esto muestra que las investigaciones se centran en la propia Inspección Educativa, y en temáticas como puede ser la supervisión educativa, la formación propia de la inspección educativa y los procedimientos de evaluación utilizados por estos profesionales en el ejercicio de sus funciones.

El nivel de productividad personal muestra que las contribuciones científicas son provenientes de un número reducido de autores, y el crecimiento exponencial muestra mayor productividad en 2011, 2014 y 2017, estando actualmente en descenso la producción científica. Finalmente, y para concluir, se marca un perfil general, en el que se determina que los textos científicos centrados en la inspección educativa son de una autoría, realizado por hombres, aplicando una metodología cualitativa, mediante el análisis de documentos, con referencias inferiores a 20 documentos, siendo el año 2017 el mayor año de producción, con artículos indexados en Latindex y creaciones de textos científicos por parte de la inspección de Andalucía, cuya palabras más usadas son "Educación" e "Inspección".

Entre las limitaciones encontradas en la elaboración del artículo se destaca la dificultad de recopilar las reseñas de la base de datos de Google Scholar, dado que no ofrece la posibilidad de descarga manual como otras bases de datos, ya sea Web of Science o Scopus. Como futuras líneas de investigación, se puede establecer un análisis de la temática de 
inspección educativa en otras bases de datos, tales como Scopus o Google Scholar, para obtener con ello una visión más amplia de las líneas de investigación desarrollada en el ámbito internacional.

\section{Referencias}

Aguerrondo, I. (2013). El rol de la supervisión educativa en la gestión de las políticas públicas. Educar, 49(1), 13-27. https://doi.org/10.5565/rev/educar.8

Aleixandre, R., González, M., González, J. y Alonso, A. (2011). Fuentes de información bibliográfica. Fundamentos para la realización de búsquedas bibliográficas. Acta Pediátrica Española, 69(3), 131-136.

Álvarez, G. (2015). La educación comparada más allá de la REEC: Análisis bibliométrico de la disciplina en las cinco revistas españolas afines de más impacto entre 1995 y 2014. Revista Española de Educación Comparada, 25, 19-45. https://doi.org/10.5944/reec.25.2015.14782

Álvarez, E. y Pérez, R. (2010). Radiografías de la inspección educativa en la comunidad autónoma de Asturias. Revisión crítica con intención de mejora. Bordón, 62(1), 9-28.

Álvarez, C., Rodríguez, M. F. y Camacho, A. (2018). Claves para el diseño de un plan de formación permanente para la inspección educativa en España. Revista de la Asociación de Inspectores de Educación de España, 29, 1-21.

Anta, C. (2008). Análisis bibliométrico de la investigación educativa divulgada en publicaciones periódicas españolas entre 1990-2002. REDIE. Revista Electrónica de Investigación Educativa, $1 O(1), 1-17$.

Antúnez, S. (2009). La inspección educativa y la evaluación de la formación permanente de los profesionales de la educación. Revista de la Asociación de Inspectores de Educación de España, $10,1-6$.

Bar, J. M. y Martínez, F. (2018). Presente y futuro de la inspección educativa. Revista de la Asociación de Inspectores de Educación de España, 30, 1-30.

Bolívar, A. (2018). La inspección educativa en un marco de autonomía escolar, una inevitable reestructuración. Fórum de Aragón, 24, 10-19.

Camacho, A. (2015). Los antecedentes remotos de la inspección educativa española durante el antiguo régimen. Supervisión21, 38, 1-14.

Carrasco, D. (2014). Organización y funcionamiento de la inspección educativa: Los equipos de zona y su adscripción. Revista de la Asociación de Inspectores de Educación de España, 21, 1-16.

Choi, A. (2019). La inspección de la educación: ¿Qué modelos funcionan mejor? Barcelona: Institut Catalá d'Avaluació de Polítiques Públiques.

Cobo, M. J., López, A. G., Herrera, E. y Herrera, F. (2011). Science mapping software tools: Review, analysis, and cooperative study among tools. Journal of the American Society for Information Science and Technology, 62(7), 1382-1402. https://doi.org/10.1002/asi.21525

Cortes, R. y Lorente, A. (2013). Los retos de la supervisión educativa en América Latina y en España. Fórum de Aragón, 8, 17-20.

Cuadrado, F. (2018). Inspección Educativa, cuasi-mercado y gobierno por los resultados. Revista de la Asociación de Inspectores de Educación de España, 30, 1-27.

Davis, J. C. y González, J. G. (2003). Scholarly journal articles about the Asian tiger economies: Authors, journals, and research fields, 1986-2001. Asian Pacific Economic Literature, 17(2), 51-61. https://doi.org/10.1046/j.1467-8411.2003.00131.x 
Espejo, L. M. y Calvo, M. I. (2015). ¿Contratendencias en la supervisión educativa? Las políticas de inclusión como herramienta de control formal en Uruguay. REICE. Revista Iberoamericana sobre Calidad, Eficacia y Cambio en Educación, 13(4), 61-78.

Esteban, S. (2010). Los últimos cuarenta años de historia de la inspección educativa en España. Revista de la Asociación de Inspectores de Educación de España,12, 1-19.

Esteban, S. (2011). Naturaleza y aportaciones de la inspección educativa como servicio público. Revista de la Asociación de Inspectores de Educación de España, 15, 1-13.

Esteban, S., Sarasúa, A., Serentill, J. y Zulueta, M. (2016). Mesa redonda: Papel de la inspección en la innovación educativa. Revista de la Asociación de Inspectores de Educación de España, 26, 121.

Estefanía, J. L. (2017). La inspección ante la innovación educativa. Revista de la Asociación de Inspectores de Educación de España, 27, 1-36.

Fernández, F. J. (2013). Objetivos de la inspección educativa de Andalucía para el cuatrienio 2012/2016. Una invitación al debate. Revista de la Asociación de Inspectores de Educación de España, 19, 1-18.

García, P. E. y Pérez, J. M. (2017). La inspección educativa como servicio público para el siglo XXI. Supervisión21, 46, 1-18.

Gómez, A., Ramiro, M. T., Ariza, T. y Reina, M. (2012). Estudio bibliométrico de Educación XXI. Educación XX1: Revista de la Facultad de Educación, 15(1), 17-41.

González, B., Moreno, L., Morillo, F. y Bordons, M. (2012). Indicadores bibliométricos para el análisis de la actividad de una institución multidisciplinar: El CSIC. Revista Española de Documentación Científica, 35(1), 9-38. https://doi.org/10.3989/redc.2012.1.851

González, E., Salmerón, A. y Beas, M. (2017). Cambios en el desempeño profesional de la inspección educativa en España en los últimos cincuenta años. Revista Linhas, 18(36), 221-250. https://doi.org/10.5965/1984723818362017221

López, L (2014). La participación de la inspección educativa en los procesos de cambio en los centros educativos. Revista de la Asociación de Inspectores de Educación de España, 22, 1-19.

Luzarraga, J. M., Núñez, J. M. y Etxeberría, J. (2018). Análisis de las expectativas de los centros de Bachillerato de alta y baja eficacia escolar. Percepción de la inspección educativa. Revista Complutense de Educación, 29(4), 1075-1090. https://doi.org/10.5209/RCED.54683

Ozkoidi, J. y Albéniz, A. (2014). Formación de la inspección educativa: Mirando al futuro. Revista de la Asociación de Inspectores de Educación de España, 22, 1-21.

Maltrás, B. (2003). Los indicadores bibliométricos: Fundamentos y aplicación al análisis de la ciencia. Madrid: Trea.

Martín, E. (1988). Supervisión educativa. Madrid: Universidad Nacional de Educación a Distancia.

Martínez, C. y Hernández, V. (2015). La evaluación de la supervisión educativa en las condiciones actuales. REICE. Revista Iberoamericana sobre Calidad. Eficacia y Cambio en Educación, 13(2), 85-98.

Moher, D., Shamseer, L., Clarke, M., Ghersi, D., Liberati, A., Petticrew, M., Shekelle, P. y Stewart, L. (2015). Preferred reporting items for systematic review and meta-analysis protocols (PRISMA-P) 2015 statement. Systematic Reviews, 4, 1-9. https://doi.org/10.1186/2046-4053-4-1

Pérez Aguilar, J. F. (2015). Instrumentos de la inspección educativa: Informes. Revista de la Asociación de Inspectores de Educación en España, 24, 1-11. 
Price, D. J. (1986). Little science, big science and beyond. Nueva York, NY: Columbia University Press.

Torres, D., Ruíz, R. y Delgado, E. (2009). Google scholar como herramienta para la evaluación científica. El Profesional Informático, 18(5), 501-510. https://doi.org/10.3145/epi.2009.sep.03

Ramírez, E. (2017). Historia de la inspección de educación en España. En E. Vázquez (Coord.), La inspección y supervisión de los centros educativos (pp. 27-60). Madrid: Universidad Nacional de Educación a Distancia.

Reyzábal, M. V. (2015). La supervisión educativa: Una profesión compleja, ética e imprescindible. REICE. Revista Iberoamericana sobre Calidad, Eficacia y Cambio en Educación, 13(4), 21-33.

Rodríguez, A. M., Trujillo, J. M. y Sánchez, J. (2019). Impacto de la productividad científica sobre competencia digital de los futuros docentes: Aproximación bibliométrica en scopus y web of science. Revista Complutense de Educación, 30(2), 623-646. https://doi.org/10.5209/RCED.58862

Secadura, T. (2011). El referente de la inspección educativa: El centro docente versus el sistema educativo. Revista de la Asociación de Inspectores de Educación de España, 15, 1-16.

Silva, B. P. (2013). El papel de la inspección escolar en la mejora de los resultados educativos. Educar, 49(1), 67-82.

Soler, E. (1993). Fundamentos de supervisión educativa. Madrid: La Muralla.

Urbizagástegui, R. (2005). La productividad científica de los autores: Un modelo de aplicación de la ley de Lotka por el método del poder inverso generalizado. Información Cultura y Sociedad, $12,51-79$.

Vázquez, E. (2018). La participación de la inspección educativa para el asesoramiento y la supervisión de la innovación escolar. International Studies on Law and Education, 29(30), 179194.

Velasco, B., Eiros, J. M. y San Román, J. A. (2012). La utilización de los indicadores bibliométricos para evaluar la actividad investigadora. Aula Abierta, 4O(2), 75-84.

Viñao, A. (1999). La inspección educativa: análisis socio-histórico de una profesión. Revista Bordón, 51(3), 251-263.

\section{Breve CV del autor}

\section{Antonio José Moreno Guerrero}

Doctor en el Departamento de Didáctica, Organización Escolar y Didácticas Especiales, dentro del Programa de Modelos Didácticos, Interculturalidad y Aplicación de las Nuevas Tecnologías en las Instituciones Educativas por la Universidad Nacional de Educación a Distancia, Licenciado en Psicopedagogía y Diplomado en Educación Especial y en Audición y Lenguaje por la Universidad de Granada. Dispone además de un Máster en informática educativa por la Universidad Nacional de Educación a Distancia. Integrante del grupo de investigación AREA (HUM-672). Autor de diversas publicaciones científicas, ponencias en distintos congresos de ámbito internacional, ponente en cursos de formación del profesorado. Miembro del equipo editorial de la Revista Científica DEDICA. Ha creado recursos educativos para editoriales y el Ministerio de Educación y Formación Profesional. ORCID: https://orcid.org/OOOO-OO03-3191-2048. Email: ajmoreno@ugr.es 